\title{
Experimental Study of Jet Vectoring by using Counter Flow at Subsonic Speed in Circular Jet
}

\author{
Ali Abdul Almuhsen Al-Asady \\ Dep. of Mechanical Engineering \\ College of Engineering \\ Baghdad University
}

\author{
Ahmed Hikmat Faseeh \\ Dep. of Mechanical Engineering \\ College of Engineering \\ Baghdad University
}

\begin{abstract}
An experimental investigation of fluidic jet vectoring using counter-flow method had been carried out in this current work. The experimental investigation included a set of experiments to examine the various effects of geometric variables on the thrust vectoring angle. These included Coanda surface radius $\mathrm{R} / \mathrm{d}=$ $(0.58823,1.17647,1.75471)$, secondary gap height $\mathrm{h} / \mathrm{d}=$ $(0.02941,0.05882)$, and secondary mass flow ratio range of $(0 \leq$ $\left.\dot{m}_{\mathrm{S}} / \dot{m}_{\mathrm{p}} \leq 0.07882\right)$. In addition, the test rig was built to display the ability of counter-flow method to vector the primary flow in pitch and yaw axes. Load cell readings were obtained using two components overhead balance. The results show proportional increase in vectoring angle value with the increase of the secondary suction rate. Three zones can be observed: a "dead zone" region at low mass flow ratios, followed by a control region where continuous primary jet control is achievable until a saturation region is reached. Small Coanda surface ratio resulted in an extended dead zone. The secondary gap height increase had an inverse effect on the thrust vectoring angle. A Smoke visualization was carried out to augment the experimental work and to demonstrate primary flow vectoring in pitch, and yaw axes. The investigation shows that the experimental gave a good agreement compared with previous studies on jet vectoring angle.
\end{abstract}

\section{Keywords}

Thrust Vectoring, Jet vectoring angle, Coanda effect, Counterflow, mass flow ratio

\section{INTRODUCTION}

An essential issue concerning performance related operation of any combat aircraft is maneuverability. A maneuver is the result of aircraft response to a control input from the pilot, and it is achieved traditionally by using aerodynamic control surfaces [1]. These surfaces are elevators, canards, rudders and ailerons. When a surface deflects, a change in the aerodynamic moments and forces is produced which causes the aircraft to maneuver [2]. These conventional control systems are inadequate due to limitation by aerodynamic constraints since whenever the aerodynamic forces are small, an effective control is either lost or compromised. In addition, the traditional control methods are heavy, complex in design and require continuous maintenance $[1,2]$. As an alternative control method, Thrust Vectoring control (TVC) has established many possible benefits to high performance aircrafts in terms of improved maneuverability, performance and survivability.

Thrust vectoring control is favorable over conventional aerodynamic control for many reasons. Fighting aircrafts' combat efficiency can be increased when multi axis thrust vectoring is added to the aircraft's propulsion system. The aircraft's ability to land and take off from short or damaged runways is also enhanced with the addition of thrust vectoring control since it provides a vertical thrust component that can be added to the lift force produced by the wings [3].

Thrust vectoring control can direct the exhaust thrust of missile or jet engine by various methods. Namely, mechanical thrust vectoring (MTV) which rely on mechanical means for vectoring or the recent methods which are fluidic-based vectoring methods (FTV). In mechanical thrust vectoring the deflection of nozzle's exhaust thrust of an aircraft is done by mechanical movements driven by pneumatic or hydraulic actuators [4]. Although these methods significantly shortened the take-off and landing distances as well as improving the maneuverability of the aircrafts, they introduced a number of disadvantages [5], since these mechanical flaps and actuators used for vectoring add additional weight and complexity to the fighter jets which in consequence increase the maintenance costs. Due to these disadvantages of the mechanical thrust vectoring, researchers sought to investigate new methods to accomplish the same thrust vectoring requirements without the use of external mechanical parts by fluidically vectoring the jet. i.e Fluidic thrust vectoring [6]

Fluidic thrust vectoring (FTV) is the use of a secondary flow source to control and redirect the exhaust jet of an aircraft without the use of movable parts. The secondary flow is taken from bleed air of an engine fan or compressor [7]. The main advantage of fluidic thrust vectoring control over its mechanical counterpart is the elimination of movable parts which substantially reduces the weight of the aircraft since large portions of the nozzle geometry could be fixed and integrated with aircraft's structure which permits the elimination of mechanical actuators and kinematic structures [8]. It also provides an enhanced stealth characteristics due to simple tail design [6]. Moreover, Fluidic vectoring techniques offer up to $80 \%$ nozzle weight reduction and $50 \%$ less maintenance costs in comparison with mechanical control schemes [9]. FTV system depends on the phenomena identified as the Coanda effect.

Coanda effect is a phenomenon discovered by and termed after the Romanian inventor and aerodynamicist Henri-Marie Coanda in 1930, in which the fluid has the inclination to attach itself to a close curved surface due to a reduction in pressure produced by the acceleration of flow around that curved surface. The attachment effect can by controlled by applying a secondary control jet to influence the main jet attachment to the wall [10]. The jet attachment remains even after the surface is curved away from its early direction which is why this effect can be used in changing the primary flow direction. The parameters that affect this phenomenon are secondary slots location and height, the surface radius of curvature, free stream velocity and the secondary stream flow ratio [11].

The fluidic jet vectoring control can be classified into five methods: Synthetic jet actuators, Co-flow, Shock vector control, Counter-flow, and Sonic throat shifting. These methods had 
been investigated numerically and experimentally with different success levels. The objective of this current work is to conduct a study of the Counter-flow vectoring method for circular nozzle exhaust. The fluidic vectoring concept was proposed by [5]. Jet deflection is accomplished by Vacuum which is generated by secondary counter flow from one of the slots located at the exit of the nozzle close to the primary jet. These slots are fitted with suction collars known as Coanda surfaces. Suction is then applied to secondary flow slot to vector the primary jet flow as an asymmetric pressure is generated by a secondary flow stream at the wall of the Coanda collar which influences the primary flow to deflect towards the low-pressure region, [6]. The basic geometry of a CFTV nozzle is illustrated in, Figure 1.

The counter-flow method was investigated experimentally in this present work. An investigation of the counter-flow jet vectoring scheme is performed to study the effect of various parameters and the design of multi axis vectoring nozzle which can be driven by a computer to control the primary jet in pitch and yaw directions. Finally, a parametric study was conducted to investigate the effects of several parameters on the jet vectoring angle, such as, Coanda wall radius of curvature (R), Secondary slot gap height (h), and mass flow ratio $\left(\dot{m}_{\mathrm{S}} / \dot{m}_{\mathrm{p}}\right)$

\section{THEORETICAL MODEL:}

\subsection{General Simplifying Assumptions:}

The two-dimensional control volume used in this study which is illustrated in Figure 2 is the same approach implemented by [1]. Using the same control volume approach as in previous studies is useful for obtaining and comparing data. In order to calculate the momentum balances a few assumptions were required since the flow properties on the boundary of the whole control volume would not all be computed. These assumptions are listed below:

1- The pressure in all boundaries of the control volume is atmospheric except in the gap and Coanda wall.

2- the momentum exiting the control volume from the nozzle is constant for each suction conditions.

3- another simplifying assumption is to assume the viscous drag is neglected since there was no a way to calculate it.

The flow is considered to be in steady state and for this condition the momentum equation is written in vector form as follows:

$\sum \vec{F}-\int \rho \vec{u}(\vec{u} \cdot \vec{n}) d A=0$

By the assumptions mentioned above, the only forces being applied in the control volume are pressure forces. The calculation of these forces is as follows:

$\sum \vec{F}-\int_{C V} \Delta p \vec{n} d A=-\int_{C V}\left(p-p_{\infty}\right) \vec{n} d A$

Since the pressure is not atmospheric at Coanda walls nor in the gap, there exist pressure forces. For the gap the normal vector is given by $\mathrm{n}=\mathrm{e}_{\mathrm{x}}$ since it has only one component parallel to the horizontal axis. As for Conada wall, the surface is curved, therefore it has two pressure force components along vertical and horizontal axis. Hence:

$\sum \vec{F}=\sum \vec{F}_{x}+\sum \vec{F}_{y}$

$\sum \vec{F}_{x}=\left(F_{\text {gap }}+F_{x \text { collar }}+R x\right) \vec{e}_{x}$

$\sum \vec{F}_{y}=\left(F_{y \text { collar }}+R y\right) \vec{e}_{y}$

As for momentum fluxes, only horizontal components are present, $-\int \rho \vec{u}(\vec{u} \cdot \vec{n}) d A=\int \rho u_{x}(\vec{u} \cdot \vec{n}) d A \vec{e}_{x}=-\left(\dot{m}_{p} u_{p}+\dot{m}_{s} u_{s}\right) \vec{e}_{x}$

when equation (3), (4) and (5) is substituted into (1) a system is obtained where it can be solved for Rx and Ry:

$$
\begin{aligned}
& F_{\text {gap }}+F_{x \text { collar }}+R x-\dot{m}_{p} u_{p}-\dot{m}_{s} u_{s}=0 \\
& F_{y \text { collar }}+R y=0 \\
& R x=\dot{m}_{p} u_{p}+\dot{m}_{s} u_{s}-F_{\text {gap }}-F_{x \text { collar }} \\
& R y=-F_{y \text { collar }}
\end{aligned}
$$

The vertical reaction force is present as a direct result vectoring the primary jet. When the jet is flowing without vectoring, only the horizonal force reactions will be present. As the vectoring angle $\delta \mathrm{v}$ starts to increase, a vertical reaction force starts to devlope. The vectoring angle value can be calculated as follow:

$$
\delta_{v}=\tan ^{-1}\left(\frac{R y}{R x}\right)
$$

\section{EXPERIMENTAL WORK 3.1 Experimental rig}

The experimental rig was built in the Heat Transfer Lab. At the Mechanical Engineering Dept. _ University of Bagdad. This experimental test rig was built to demonstrate to ability to divert the direction of a main exhaust flow in two axes (pitch and yaw) by the control of a secondary counter flow. A schematic diagram is shown in Figure 3. The test section shown in Figure 4a consists of a circular duct made from aluminum tube with an outer diameter of $38 \mathrm{~mm}$ and an inner diameter of $\mathrm{d}=34 \mathrm{~mm}$ and length of $\mathrm{L}=750 \mathrm{~mm}$. This duct is attached to a centrifugal blower by flexible hose at one end and at the other end is fitted with a peripheral cylinder that introduces the secondary flow. This cylinder has a $73 \mathrm{~mm}$ outer diameter and consists of four separate equal channels surrounding the circular duct and parallel to the main flow direction as shown in Figure 4b-c. The four inlets have a constant slot height of $2 \mathrm{~mm}$. The channels outlets are attached to the ejector by four flexible hoses. Furthermore, this cylinder is used to mount different compatible curved collars (Coanda surfaces) to utilize the Coanda effect for fluidic jet vectoring.

Six different collar configurations were studied to examine the effects of changing the collar radius of curvature on the jet vectoring angle. The main dimensions are radius of curvature $\mathrm{R}=20 \mathrm{~mm}, 40 \mathrm{~mm}$ and $60 \mathrm{~mm}(\mathrm{R} / \mathrm{d}=0.58823,1.17647$, and 1.76471) for two different secondary slot heights $\mathrm{h}=1 \mathrm{~mm}$, and $2 \mathrm{~mm}(\mathrm{~h} / \mathrm{d}=0.02941,0.05882)$. These collars are also made of aluminum with a length of $20 \mathrm{~mm}$ and $30 \mathrm{~mm}$, outer diameter of $D o=73 \mathrm{~mm}$ and inner diameter of $\mathrm{D}_{\mathrm{i}}=(40 \mathrm{~mm}, 42 \mathrm{~mm})$ respectively for the two slot heights. Moreover, the experimental test included ten secondary air mass flows for every collar for the specific main jet velocity. A centrifugal blower with flow rate of $11 \mathrm{~m}^{3} / \mathrm{min}$ was suitable to provide the main jet for the selected outflow velocities. An ejector pump was used as vacuum source for the secondary counter flow. The load cells used were selected to withstand the test section weight and have the sensitivity to measure the jet reaction forces to calculate the vectoring angles. By placing the Coanda collar at the outlet of the primary nozzle by two screws to the peripheral cylinder and starting a secondary suction air stream, parallel to the Coanda collar wall in the opposite direction of the main flow a counterflow fluidic jet vectoring can be obtained. The jet vectoring angle obtained can be calculated from equation (11). 


\subsection{Multi axis vectoring}

The secondary flow direction is controlled by two modified directional valves. The valves are controlled by two servo motors and an Arduino board. The Arduino is an open-source modeling board based on the ATmega328P microcontroller which can be programmed by a computer integrated development environment (IDE). Arduino boards are capable of reading inputs from sensors, button, serial ports and in turn controlling an output such as motors, lights, relays and other actuators. The Arduino board used in this work was an Arduino UNO R3. It was used to control two servo motors to operate two directional valves, as shown in Figure 3 and Figure 4a. The board was interfaced with the computer through serial port and received its order from a computer program by using the Python programming language. A schematic of the Arduino board valve control is illustrated in Figure 5.

\section{ERROR ANALYSIS}

The precision of obtained experimental results depends on two factors. The design details of the test rig and the accuracy of measurements. The deviations in accuracy are resulted from;

1. The uniformities in air flow.

2. The alignment of fixing load cell.

3. Instrument for measuring the flow.

Let the result $\mathrm{R}$ be a function of (n) independent variables $\left(\mathrm{v}_{1}\right.$, $\left.\mathrm{v}_{2} \ldots \mathrm{v}_{\mathrm{n}}\right)$

$\left.\mathrm{R}=\mathrm{R}\left(\mathrm{v}_{1}, \mathrm{v}_{2} \ldots \mathrm{v}_{\mathrm{n}}\right)\right)$

For slight differences in the variables, this relationship can be stated in linear form Holman [15] as:

$\delta R=\frac{\partial R}{\partial v_{1}} \delta v_{1}+\frac{\partial R}{\partial v_{2}} \delta v_{2}+\cdots+\frac{\partial R}{\partial v_{n}} \delta v_{n}$

Hence, the uncertainty intervals (w) in the result can be given as

$\mathrm{W}_{\mathrm{R}}=\left[\left(\frac{\partial \mathrm{R}}{\partial \mathrm{v}_{1}} * \mathrm{w}_{1}\right)^{2}+\left(\frac{\partial \mathrm{R}}{\partial \mathrm{v}_{2}} * \mathrm{w}_{2}\right)^{2}+\cdots+\left(\frac{\partial \mathrm{R}}{\partial \mathrm{v}_{\mathrm{n}}} * \mathrm{~W}_{\mathrm{n}}\right)^{2}\right]^{0.5}$

Eq. (14) is greatly simplified upon dividing by Eq. (12) to non dimensionalise

$\frac{\mathrm{w}_{\mathrm{R}}}{\mathrm{R}}=\left[\left(\frac{\partial \mathrm{R}}{\partial \mathrm{v}_{1}} * \frac{\mathrm{w}_{1}}{\mathrm{R}}\right)^{2}+\left(\frac{\partial \mathrm{R}}{\partial \mathrm{v}_{2}} * \frac{\mathrm{w}_{2}}{\mathrm{R}}\right)^{2}+\cdots \cdots+\left(\frac{\partial \mathrm{R}}{\partial \mathrm{v}_{\mathrm{n}}} * \frac{\mathrm{w}_{\mathrm{n}}}{\mathrm{R}}\right)^{2}\right]^{0.5}$

Therefore, for the variables used the possible experimental errors are given in $(0.001 \mathrm{~g})$ which is taken from the weight indicator and load cell Now;

Thrust vectoring angle $(\delta)=\tan ^{-1} \frac{R_{y}}{R_{x}}$

$\frac{\partial \delta}{\partial R_{y}}=\frac{1}{1+\left(\frac{R_{y}}{R_{x}}\right)^{2}} * \frac{1}{R_{x}}$

$\frac{\partial \delta}{\partial R_{x}}=\frac{1}{1+\left(\frac{R_{y}}{R_{x}}\right)^{2}} * \frac{-R_{y}}{R_{x}^{2}}$

Therefore, the uncertainty intervals (w) in the result can be given as follows;

$w_{\delta}=\left[\left(\frac{\partial \delta}{\partial R_{y}} \cdot w_{R_{y}}\right)^{2}+\left(\frac{\partial \delta}{\partial R_{x}} \cdot w_{R_{x}}\right)^{2}\right]^{0.5}$.

So, $(\delta)=\tan ^{-1} \frac{R_{y}}{R_{x}}=\tan ^{-1}(.0168 / 0.038)=23.85^{\circ}$ $\frac{\partial \delta}{\partial R_{y}}=\frac{1}{1+\left(\frac{0.0168}{0.038}\right)^{2}} * \frac{1}{(0.038)}=22.01316$

$\frac{\partial \delta}{\partial R_{x}}=\frac{1}{1+\left(\frac{0.0168}{0.038}\right)^{2}} * \frac{-0.0168}{0.038^{2}}=-9.73$

$w_{\delta}=\left[(22.01316 \times 0.001)^{2}+(-9.73 * 0.001)^{2}\right]^{0.5}=$ 0.02406

$\frac{\mathrm{w}_{\delta}}{\delta}=\frac{0.02406}{23.85}=0.101 \%$

\section{RESULTS AND DISCUSSION}

A series of tests were made in order to examine the effects of geometry variables and flow conditions on the jet vectoring angle. These tests included varying Secondary slot height (h), Coanda surface radius of curvature $(\mathrm{R})$ and mass flow ratio $\left(\dot{m}_{\mathrm{S}} / \dot{m}_{\mathrm{p}}\right)$

\subsection{Varying Secondary Gap Height $(h / d)$ :}

Figure 6 shows the relationship between the resulting jet vectoring angle and the mass flow ratio $\dot{m}_{\mathrm{S}} / \dot{m}_{\mathrm{p}}$ for two secondary gap heights at a constant Coanda surface of ratio R/d $=0.58823$. The gap heights tested were $\mathrm{h} / \mathrm{d}=0.02941$ and $\mathrm{h} / \mathrm{d}=$ 0.05882 for the primary flow velocity $\mathrm{Vp}=20 \mathrm{~m} / \mathrm{s}$. The results show a decrease in the vectoring angle value as the secondary gap height is increased for each mass flow ratio tested. Since the gap height determines the area open for the counter flow stream, increasing the gap height will increase the cross-sectional area of the secondary slot which means a lower secondary velocity for the same mass flow. This increase will yield a lesser secondary slot and collar wall pressure difference with the atmosphere than that of smaller gap heights which leads to less vectoring angle values.

It can also be seen that the thrust vectoring angle value rises as the secondary suction rate is increased. The reason behind this behavior is as the suction flow applied is increased two resulting effects take place at once, the gap pressure decreases which leads to higher secondary flow velocity, and the jet deflection towards the collar walls causes a decrease in the secondary flow area. Figure 7 and 8 show the relationship between and the thrust vector angle and $\dot{m}_{\mathrm{S}} / \dot{m}_{\mathrm{p}}$ at constant Coanda surface radius in the range of $\mathrm{R} / \mathrm{d}=1.17647,1.76471$ respectively. These results show that as the secondary suction flow rate is increased, the thrust vectoring angle value increases. It can be seen that lower vectoring angles values were obtained for the higher secondary gap height at each mass flow ratio tested. In addition, Figure 6-8 show that for a smaller gap height the required counter flow is less for the same vectoring angle value which means the attachment can occur for lower amounts of suction levels.

In Figure 6-8 The results showed that at very low secondary suction rates $\dot{m}_{\mathrm{S}} \ll \dot{m}_{\mathrm{p}}$, the vectoring angles formed were very small which developed what appears to be a "dead zone" where adequate flow control cannot be achieved. This condition can be based on the fact that the Coanda effect cannot function at low secondary suction rates because at low suction rates, an early separation of secondary jet from the Coanda wall occurs. Faster flowing primary jet will entrain the slower secondary flow which rather than adhering to the Coanda collar wall and remain attached farther downstream separates and generates a higherpressure region. After the "dead zone", Coanda effect takes over and the curve enters a region where greater thrust vectoring angles can be obtained for moderately smaller increases in the mass flow rates. This region insures a continuous control of the primary jet until the thrust vectoring angle and consequently the thrust vector force produced will arrive at a hypothetical region of saturation where the thrust vector angle reaches a nearly 
constant value, for any increase in the mass flow ratio. This trend is shown schematically in Figure 9.

\subsection{Varying Coanda Surface Radius (R/d):}

The resultant thrust vectoring angles have been obtained at various mass flow ratios $\left(\dot{m}_{\mathrm{S}} / \dot{m}_{\mathrm{p}}\right)$ for three Coanda wall radii. Figure. 10 and 11 represent the relationship between thrust vectoring angle and $\dot{m}_{\mathrm{S}} / \dot{m}_{\mathrm{p}}$ for constant gap height of $\mathrm{h} / \mathrm{d}=$ 0.02941 and $\mathrm{h} / \mathrm{d}=0.05882$ at different Coanda surface radius $\mathrm{R} / \mathrm{d}=0.58823,1.17647$, and 1.76471 for primary flow velocity $\mathrm{Vp}=20 \mathrm{~m} / \mathrm{s}$. The results showed that as the mass flow rate ratio is increased, the thrust vector angle value increases. And as can be seen from Figure 10 and 11, the thrust vectoring angle value increases proportionally with the increase of Coanda wall radius for each mass flow ratio tested because increasing collar length enlarges the area on the which the gauge collar pressure distribution can act upon. Larger radii of Coanda collars provide larger surfaces for the fluid to attach to and the generated pressure difference is higher than that generated from smaller Coanda radius which in turn increases the normal collar force and as a result, a greater thrust vectoring angle can be achieved. Also, with the increase of Coanda surface radius the slope of the curve becomes steeper once it overcomes the "dead zone". The Coanda surface radius affects the size of the "dead zone" as well, and as a consequence where the control region begins. The results showed for smaller mass flow ratios the jet is more likely to separate for a smaller radius of Coanda surface and therefore the "dead zone" will be extended over a wider range of secondary suction rate values.

\section{SMOKE FLOW VISUALIZATION}

Figure 12a shows the visualization of a non-vectored primary jet for $\mathrm{R} / \mathrm{d}=1.174647, \mathrm{~h} / \mathrm{d}=0.02941, \dot{m}_{s} / m_{p}=0$. Figure $12 \mathrm{~b}$-e show the visualization of a vectored primary jet in pitch and yaw axes for Coanda surface radius ratio of $\mathrm{R} / \mathrm{d}=1.174647$ and $\dot{m}_{\mathrm{S}} / \dot{m}_{\mathrm{p}}=0.06568$ at constant secondary gap height ratio of $\mathrm{h} / \mathrm{d}=$ 0.02941 . The smoke visualization in Figure 12 demonstrated that vectoring control was achieved by controlling the secondary slot flow automatically using a directional valve controlled by the Arduino control board that receives its orders from computer interface to simulate a flight envelope.

\section{VERIFICATION OF RESULTS}

A comparison was made with the results achieved from previous studies to verify the results obtained from the present study. The present results for the effect of varying secondary gap height on thrust vectoring angle at constant Coanda surface radius shown in Figure 6-8 are in good agreement with the results of (the experimental and numerical work) of [14] shown in Figure 13, and with (the experimental and numerical work) of [12] shown in Figure 14.

The present results for the effect of varying Coanda surface ratio on thrust vectoring angle at constant secondary gap height shown in Figure 10 and Figure 11 agree with the following previous works (the experimental and numerical work [13] shown in Figure 15 and with (the experimental and numerical work [14] shown in Figure 16

\section{CONCLUSIONS}

Fluidic jet vectoring using counter-flow method at subsonic flow have been investigated experimentally in this present work. The present study showed a considerable effect of Coanda wall radius, secondary gap height, and the mass flow ratio on the jet vectoring angle characteristics. These are concluded as follows:

- Three regions can be observed from the relationship relating jet vectoring angle and mass flow ratio. A 'dead zone' appeared at low secondary mass flowrates where the thrust vectoring angle measured was small and an adequate thrust vectoring control was not possible. Followed by an active control region in which a continuous jet vectoring control can be achieved. Last, a hypothetical saturation region is then formed where the thrust vectoring angle value nearly remained constant for any further increase in secondary suction rate.

- The vectoring angle value was increased by increasing the mass flow ratio, and Coanda surface radius once the dead zone has been overcome.

- The size of radius of curvature of the Coanda surface determined the length of the dead zone. A small surface radius resulted in an extended region of the dead zone.

- The secondary gap height has an inverse effect on the thrust vectoring angle. The thrust vectoring angle was decreased by increasing the secondary gap height for each mass flow ratio tested.

- An effective multi-axis control of the primary jet was possible for the current exit nozzle design which can be prominent for future development of fluidic multi-axis vectoring nozzles.

- Vectoring control was performed automatically using directional valves controlled by an Arduino Uno board through computer interface to simulate a flight envelope which was demonstrated by smoke visualization.

\section{REFERENCES}

[1] M. M. Santos, "Experimental Study on Counterflow Thrust Vectoring of a Gas Turbine Engine," Electronic Theses, Treatises and Dissertations. Paper 2080, 2005.

[2] T. M. Ragab and B. Elhadidi, "Counter Flow Fluid Thrust Vector Applied to Small Business Jets,” pp. 1-13, 2009.

[3] J. D. Flamm, "Experimental Study of a Nozzle Using Fluidic Counterflow for Thrust Vectoring," Control, 1998.

[4] D. Ikaza, "THRUST VECTORING NOZZLE FOR MILITARY AIRCRAFT ENGINES,” pp. 1-10, 2000.

[5] P. J. Strykowski, and A. Krothapalli, "Vectoring Thrust Using Confined Shear Layers," Naval Research Reviews, vol. 51, no. 3, pp. 26_34, 1999.

[6] L. Li, M. Hirota, and T. Saito, "Numerical and Experimental Studies of Fluidic Thrust Vectoring," 28th Int. Symp. Shock Waves, pp. 2-7, 2011.

[7] K. A. Deere, B. L. Berrier, J. D. Flamm, and S. K. Johnson, "A computational study of a new dual throat fluidic thrust vectoring nozzle concept," AIAA Paper., vol. 3502, 2005.

[8] D. J. Wing and V. J. Giuliano, "Fluidic Thrust Vectoring of an Axisymmetric Exhaust Nozzle at Static Conditions," 1997.

[9] K. Deere, "Summary of fluidic thrust vectoring research conducted at NASA langley research center," 21st AIAA Appl. Aerodyn. Conf. AIAA. Conference Paper, June, pp. 1-18, 2003.

[10] C. Olivotto, "Fluidic Elements based on Coanda Effect," vol. 2, no. 4, pp. 163-172, 2010.

[11] "CoandaEffect" https://www.thermofluids.co.uk/effect.php. [website]: [website] 
[12] A. Banazadeh and F. Saghafi, "Experimental and computational investigation into the use of co-flow fluidic thrust vectoring on a small gas turbine," no. 3117 , pp. $17-$ 25,2008

[13] Hatem H. Saleh and Dr. Ali Alassadi, “ Theoretical and Experimental Study of Fluidic Jet Vectoring in Subsonic Flow for Circular Nozzle using Co-flow Method," - M.Sc Thesis, Baghdad: University of Baghdad / College of Engineering, 2015.

[14] M. S. Mason and W. J. Crowther, "Fluidic thrust vectoring of low observable aircraft," in CEAS Aerospace Aerodynamic Research Conference, 2002, pp. 1-7.

[15] Holman, J. P. " Experimental Method For Engineers", McGraw-Hill,Tokyo, Japan, 4th Edition (1984)

\section{NOMENCLATURE}

\begin{tabular}{|l|l|l|}
\hline Symbol & Definition & Units \\
\hline $\mathrm{d}$ & Diameter of Primary Jet & $\mathrm{mm}$ \\
\hline $\mathrm{h}$ & Secondary Gap Height & $\mathrm{mm}$ \\
\hline$\dot{m}_{\mathrm{p}}$ & Primary Mass flow rate & $\mathrm{Kg} / \mathrm{s}$ \\
\hline$\dot{m}_{\mathrm{S}}$ & Secondary Mass Flow Rate & $\mathrm{Kg} / \mathrm{s}$ \\
\hline $\mathrm{R}$ & Conda surface Radius of curvature & $\mathrm{mm}$ \\
\hline $\mathrm{Re}$ & Renolds number & \\
\hline $\mathrm{Rx}$ & Horizontal reaction Force & $\mathrm{kg}$ \\
\hline $\mathrm{Ry}$ & Vertical reaction Force & $\mathrm{kg}$ \\
\hline$\delta_{\mathrm{v}}$ & Jet vectoring angle & $\mathrm{degree}$ \\
\hline$\rho$ & Density & $\mathrm{Kg} / \mathrm{m}^{3}$ \\
\hline FTV & Fluidic thrust vectoring & \\
\hline CFTV & Counter flow thrust vectoring & \\
\hline MTV & Mechanical thrust vectoring & \\
\hline $\mathrm{u}$ & Velocity component & $\mathrm{m} / \mathrm{s}$ \\
\hline
\end{tabular}

\begin{tabular}{|l|l|l|}
\hline $\mathrm{Vp}$ & Velocity of the primary flow & $\mathrm{m} / \mathrm{s}$ \\
\hline$w_{\delta}$ & uncertainty interval & \\
\hline$\Delta p$ & gage pressure difference & $\mathrm{Pa}$ \\
\hline
\end{tabular}

\section{APPENDIX}

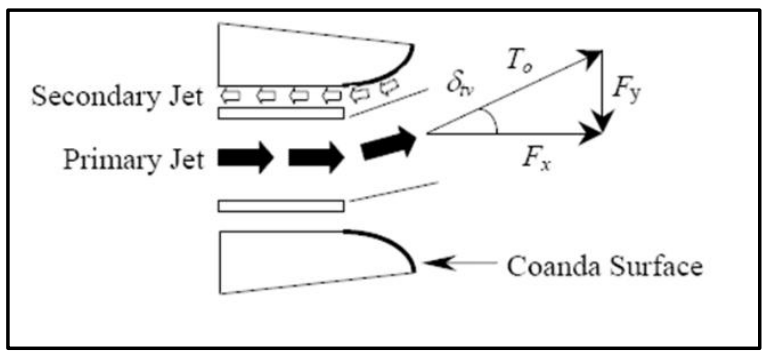

Figure 1: Counter-flow jet vectoring concept used in present work [9]

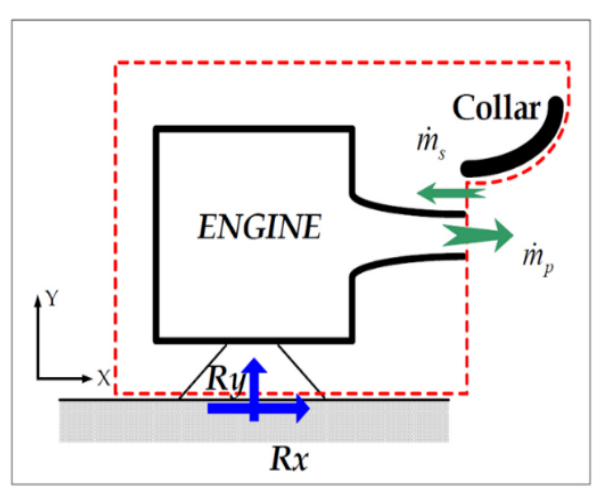

Figure 2: Control volume used for jet vectoring estimation бv

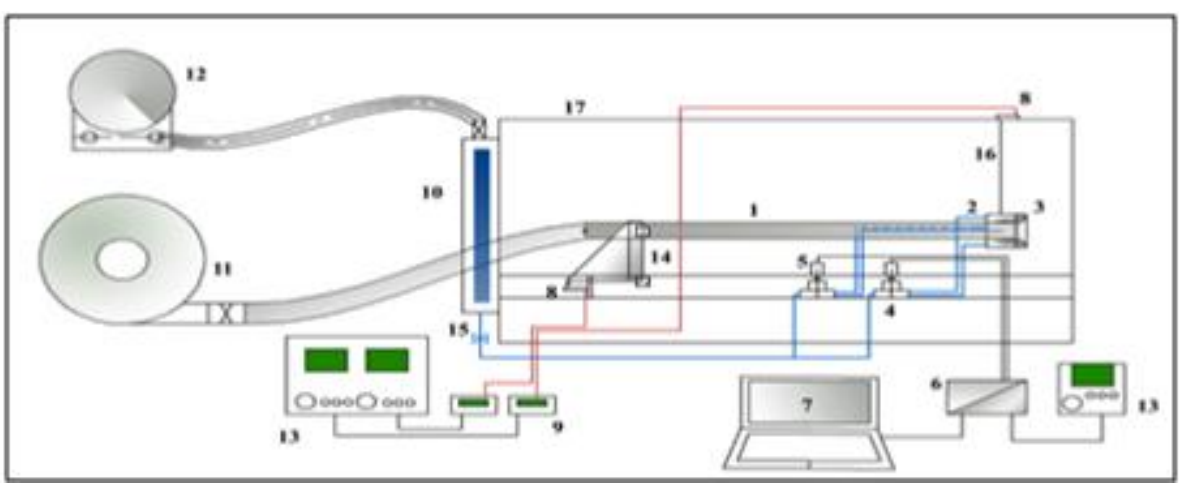

\begin{tabular}{|l|l|l|l|l|l|}
\hline 1 & Primary Duct (Circular & 7 & Laptop & 13 & Power Supply (2) \\
\hline 2 & Secondary flow pipes & 8 & Load Cells (2) & 14 & $1: 1$ lever and ball \\
\hline 3 & Coanda Collar & 9 & Weight indicator (2) & 15 & Needle valve \\
\hline 4 & Directional Valve (2) & 10 & Air Flow meter & 16 & Chain link \\
\hline 5 & Servo motor (2) & 11 & Centrifugal Blower & 17 & Frame \\
\hline 6 & Arduino Board Control & 12 & Ejector & & \\
\hline
\end{tabular}

Figure 3: Diagram of the experimental rig 


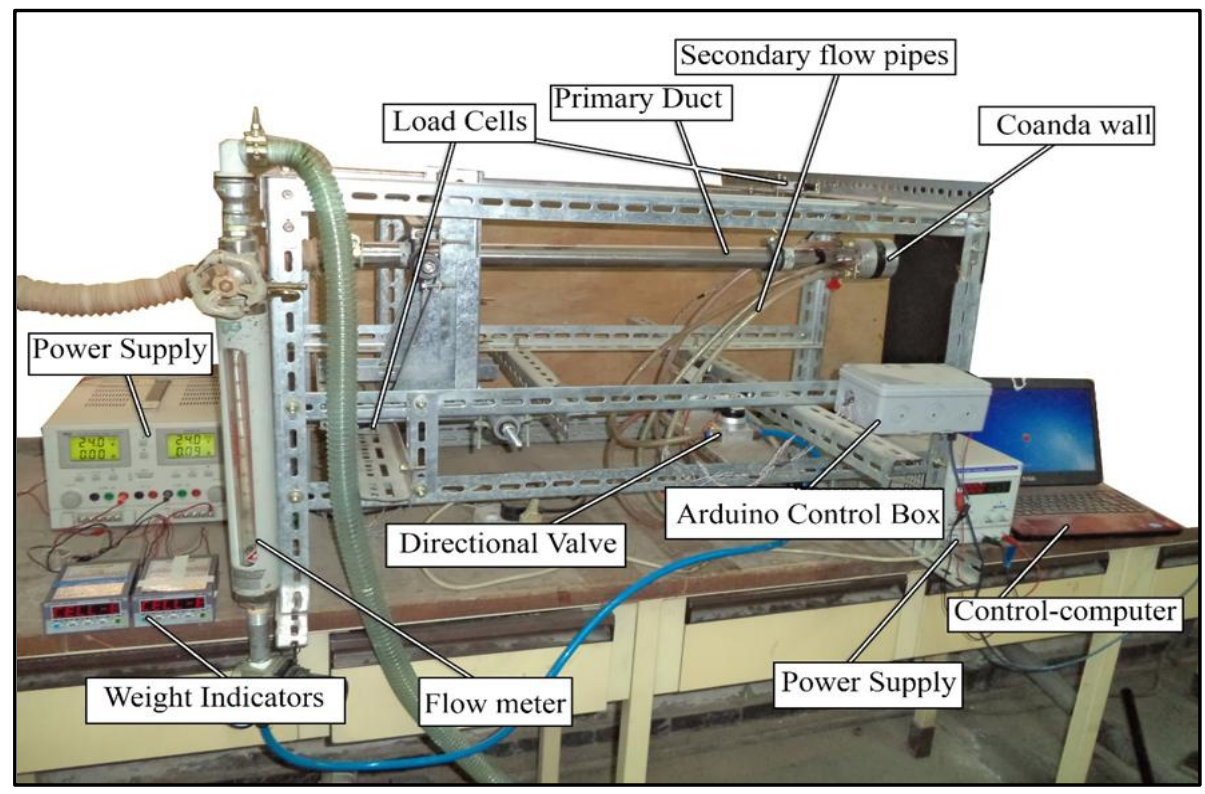

A- Photograph of the experimental rig

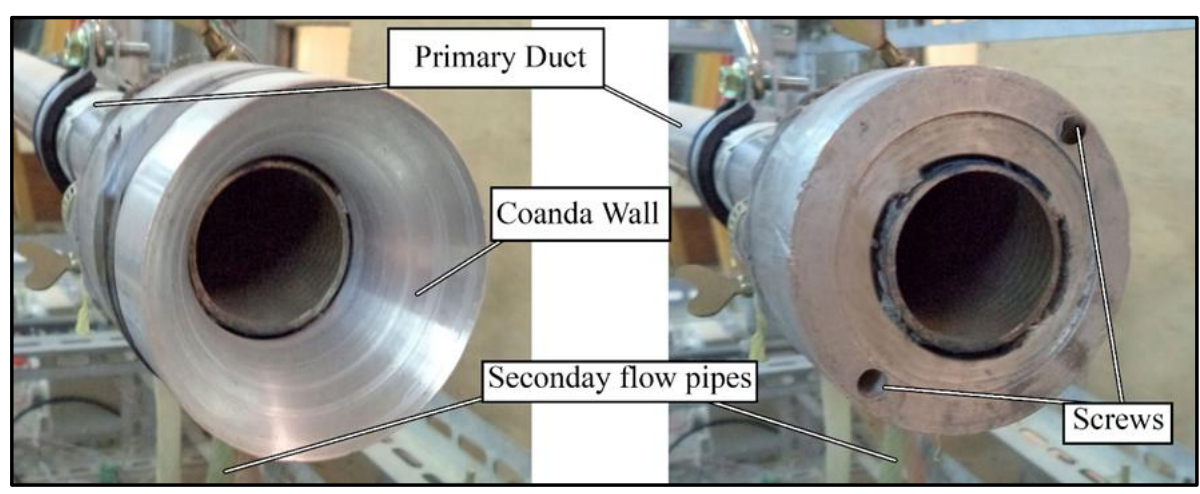

B- Nozzle exit with Coanda wall

C- Nozzle exit without Coanda wall

Figure 4: Nozzle exit and Coanda surfaces collars

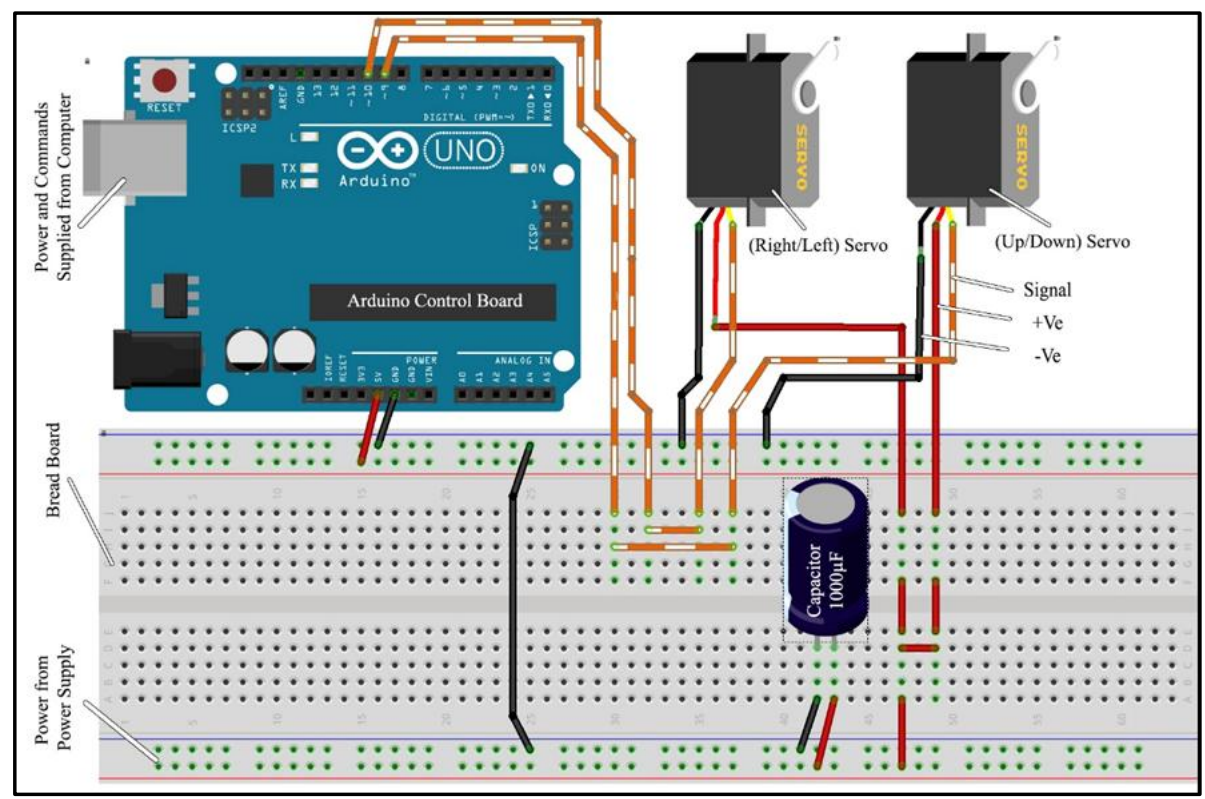

Figure 5: Schematic of the Arduino board valve control circuit 


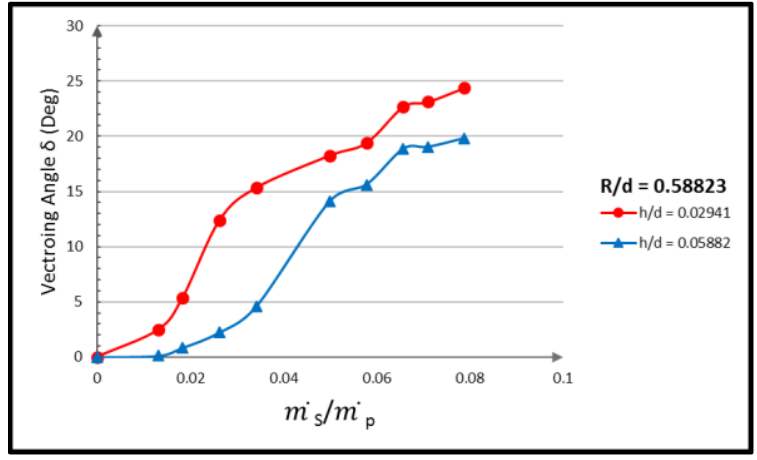

Figure 6: Experimental results of Thrust vectoring angle for varying secondary gap height at constant Coanda surface radius $R / d=\mathbf{0 . 5 8 8 2 3}$

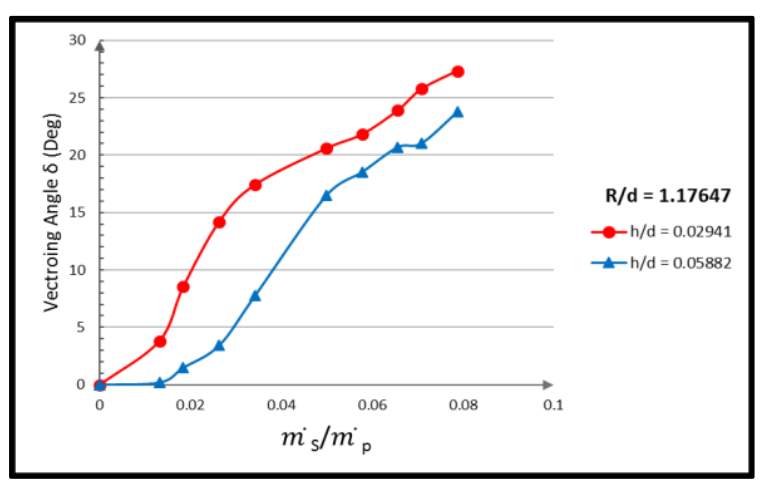

Figure 7: Experimental results of Thrust vectoring angle for varying secondary gap height at constant Coanda surface radius $R / d=1.17647$

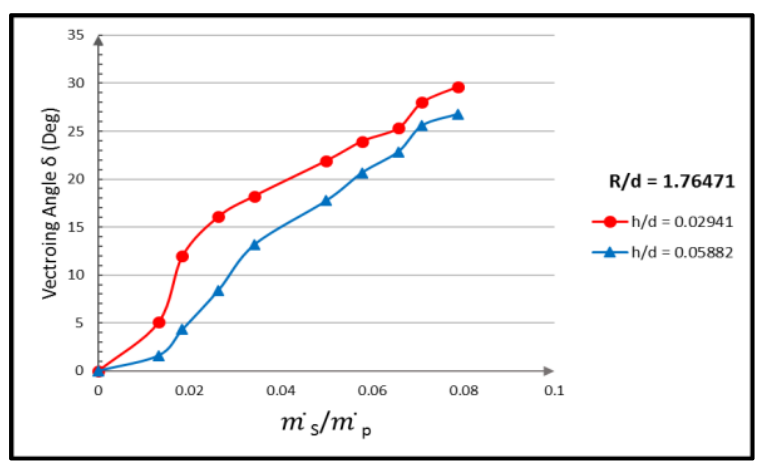

Figure 8: Experimental results of Thrust vectoring angle for varying secondary gap height at constant Coanda surface radius $R / d=1.76471$

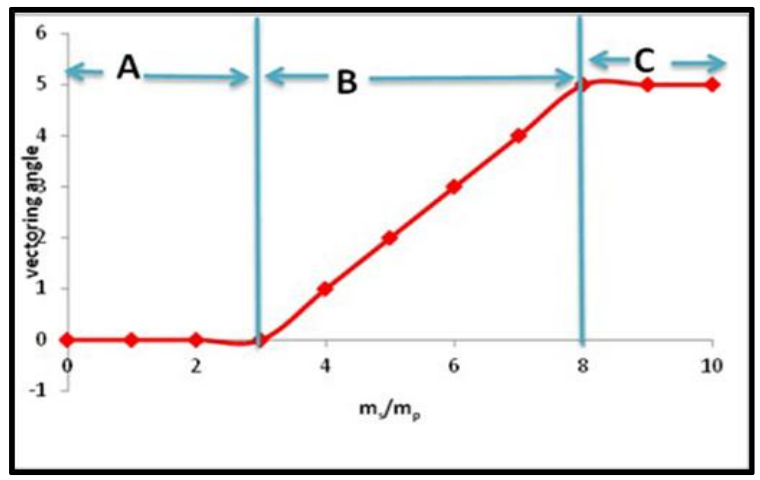

Figure 9: Trend line of the experimental results

A - Dead zone, B - Active control, C - Saturation

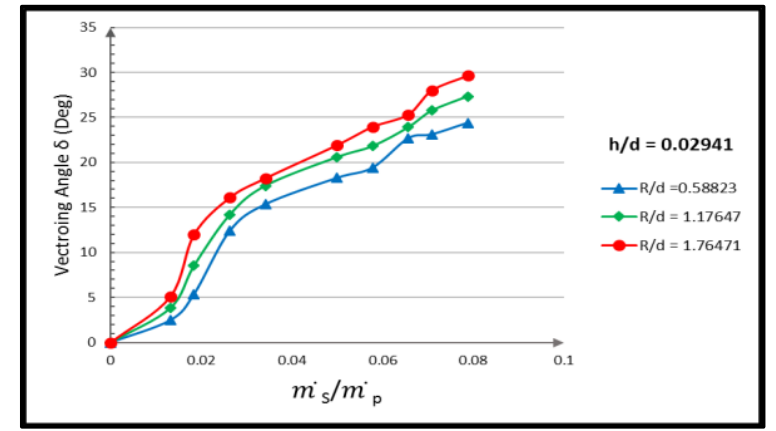

Figure 10: Experimental results of Thrust vectoring angle for varying Coanda surface radius at constant secondary gap height $\mathbf{h} / \mathbf{d}=\mathbf{0 . 0 2 9 4 1}$

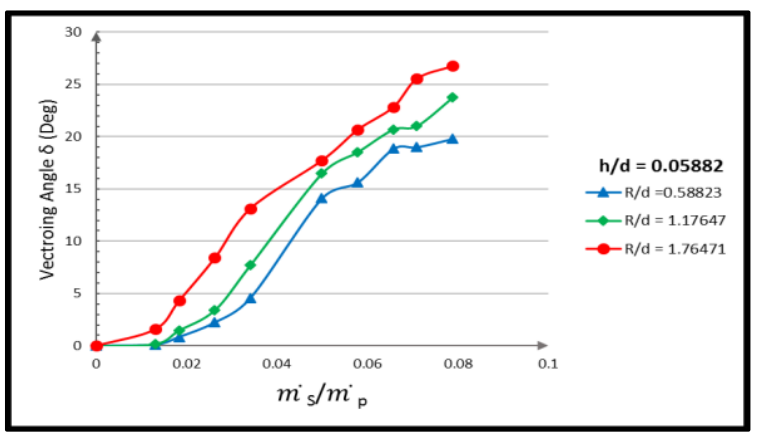

Figure 11: Experimental results of Thrust vectoring angle for varying Coanda surface radius at constant secondary gap height $\mathbf{h} / \mathrm{d}=\mathbf{0 . 0 5 8 8 2}$

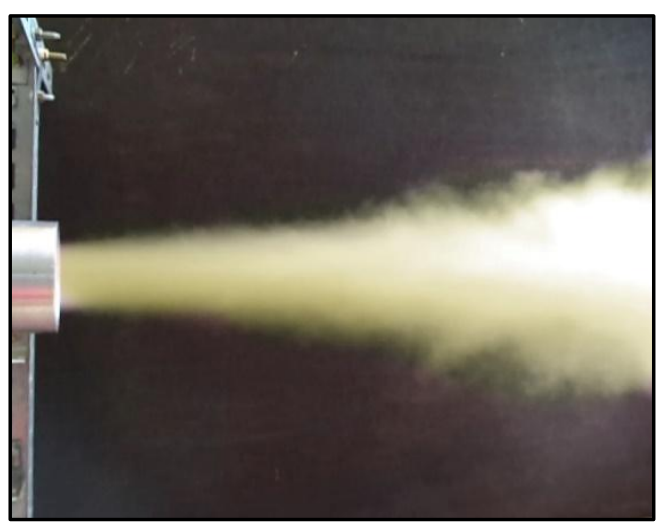

(a) Non-vectored primary flow, $R / d=1.174647, \mathrm{~h} / \mathrm{d}=$ 0.02941, $\dot{\boldsymbol{m}}_{s} / \dot{\boldsymbol{m}}_{\boldsymbol{p}}=0$

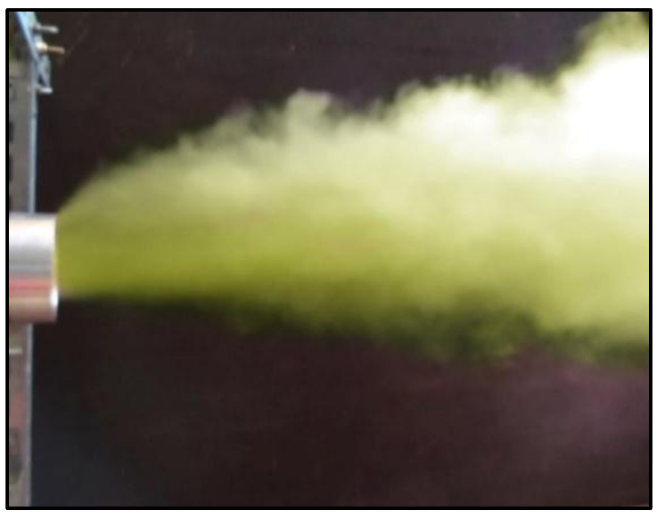

(b) Positive pitch vectoring

$\mathrm{R} / \mathrm{d}=1.174647, \mathrm{~h} / \mathrm{d}=0.02941, \dot{m}_{s} / \dot{m}_{p}=0.06568$ 


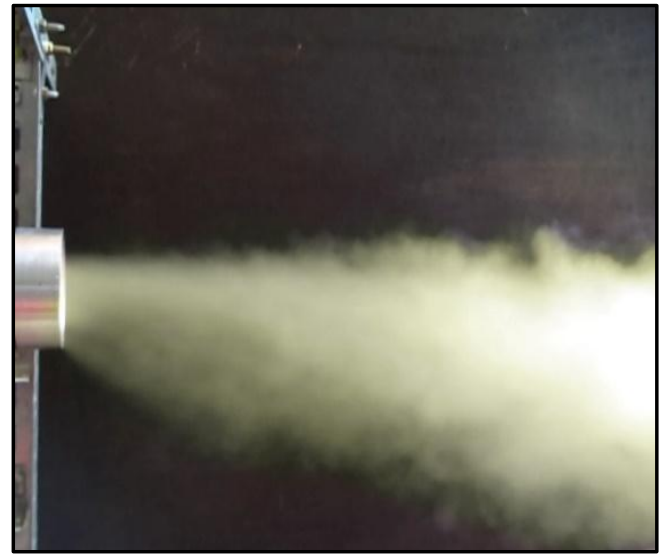

(c) Negative pitch vectoring

$\mathrm{R} / \mathrm{d}=1.174647, \mathrm{~h} / \mathrm{d}=\mathbf{0 . 0 2 9 4 1}, \dot{\boldsymbol{m}}_{s} / \dot{\boldsymbol{m}}_{\boldsymbol{p}}=\mathbf{0 . 0 6 5 6 8}$

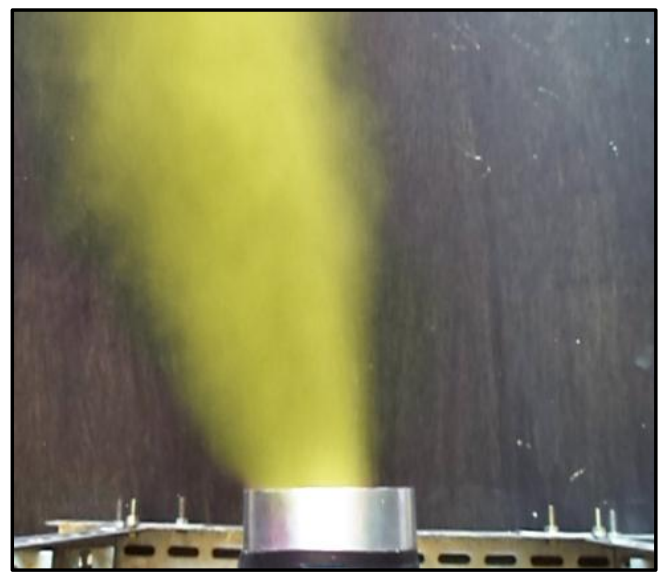

(d) Positive yaw vectoring

$\mathrm{R} / \mathrm{d}=\mathbf{1 . 1 7 4 6 4 7}, \mathrm{h} / \mathrm{d}=\mathbf{0 . 0 2 9 4 1}, \dot{\boldsymbol{m}}_{s} / \dot{m}_{p}=\mathbf{0 . 0 6 5 6 8}$

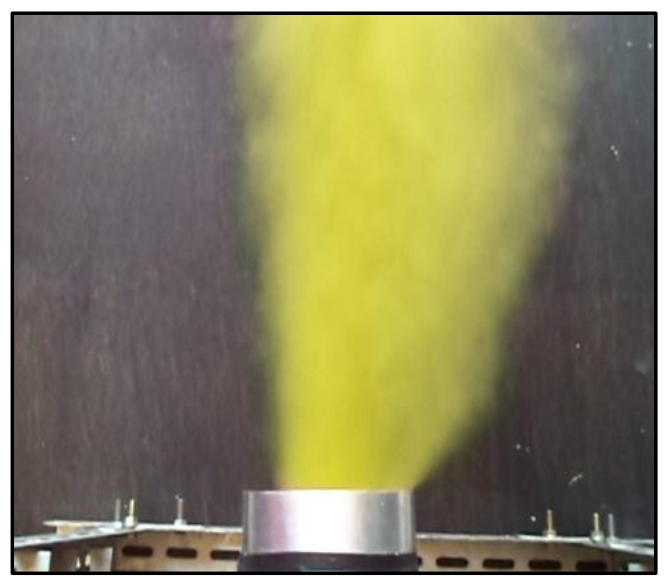

(e) Negative yaw vectoring

$\mathrm{R} / \mathrm{d}=1.174647, \mathrm{~h} / \mathrm{d}=\mathbf{0 . 0 2 9 4 1}, \dot{m}_{s} / \dot{m}_{p}=0.06568$

Figure 12: Smoke visualization of a vectored and nonvectored primary jet

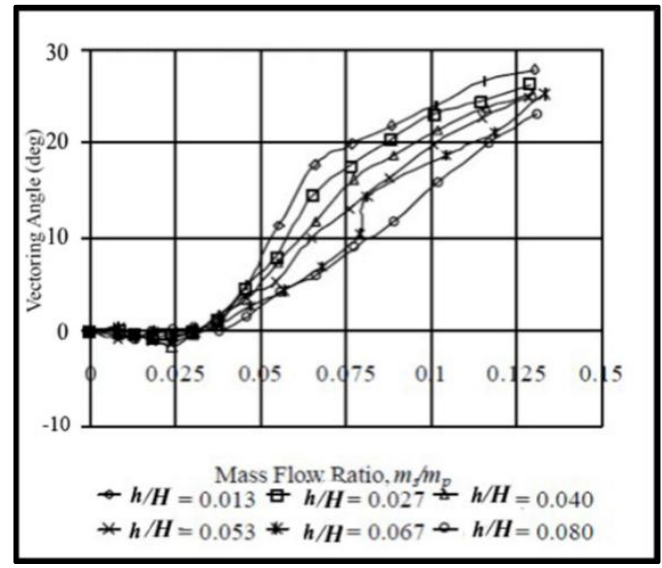

Figure 13: Experimental results [14]

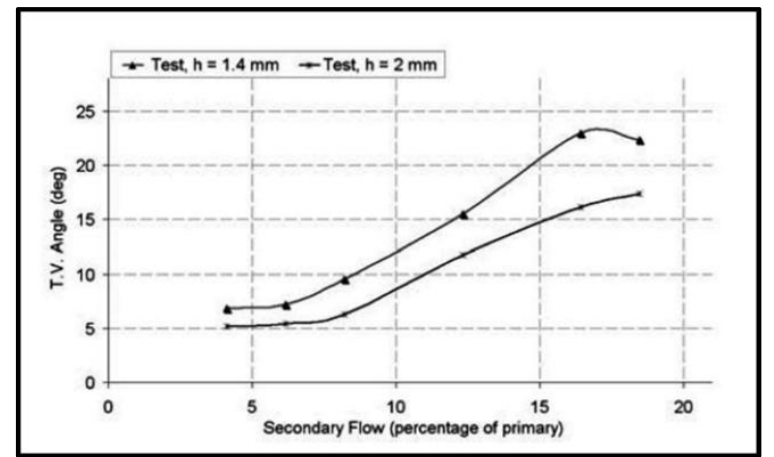

Figure 14: Experimental results [12]

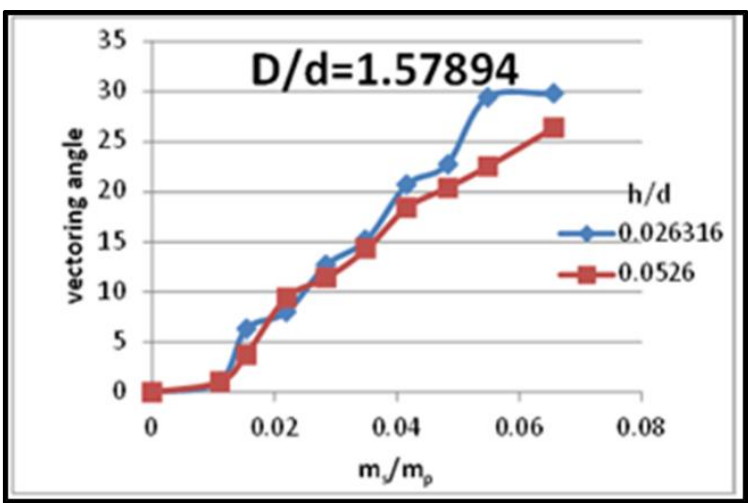

Figure 15: Experimental results [13]

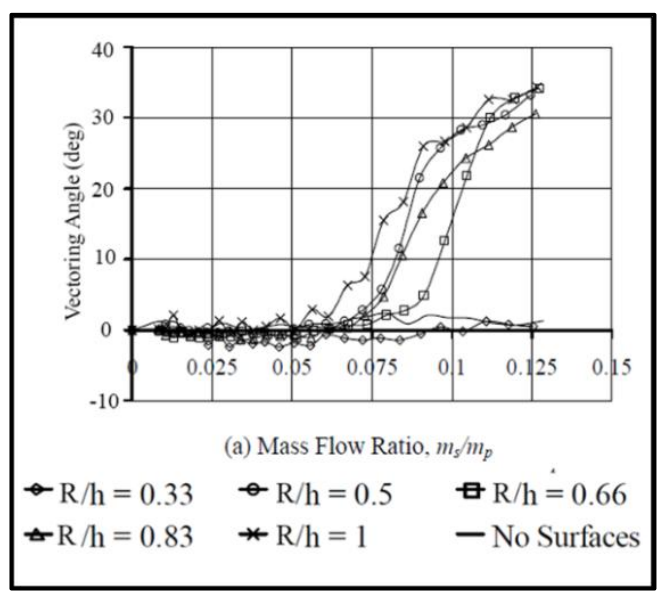

Figure 16: Experimental results [14] 